\title{
N-Way Microwave Power Divider Using Two-Dimensional
}

\section{Meta-Materials}

\section{Author:}

K. W. Eccleston

\section{Author Affiliation:}

Dept of Electrical and Computer Engineering

University of Canterbury

Christchurch

New Zealand

Email: kim.eccleston@elec.canterbury.ac.nz

\begin{abstract}
An N-way power divider based upon meta-materials is presented. The divider essentially comprises a two-dimensional mosaic of both right-handed and lefthanded four-port unit cells. The power divider is square shaped and this allows ease of integration with amplifiers. The feasibility of the proposed divided is demonstrated by the simulation of a 20 -way divider operating at $1 \mathrm{GHz}$.
\end{abstract}

\section{Introduction:}

The radial power divider is well known for its ability to realise $\mathrm{N}$-way power division for large values of $\mathrm{N}$. This ability stems from the circular symmetry of the radial waveguide, but careful design is required to ensure spurious modes are not excited [1]. In recent years, meta-materials that display left-handed propagation behaviour have 
been extensively investigated and applied to microwave devices including hybrids and power dividers [2] - [6]. These devices have all been based upon one-dimensional lefthanded and right-handed transmission lines. The propagation properties of twodimensional left-handed transmission structures have been investigated [7]. These investigations have been largely focussed on the investigation of the negative refraction phenomena encountered at the interface of two-dimensional right-handed and twodimensional left-handed regions [7]. Moreover, there are distinct left-handed and righthanded regions. In this letter we propose an N-way power divider comprising a twodimensional meta-material mosaic of left-handed and right-handed unit cells.

\section{Principle of Operation:}

The basic elements of the divider are transmission lines that exhibit left-handed behaviour and are denoted LTL, and those that are right-handed RTL. Both types of transmission lines have the same characteristic impedance (or Bloch impedance for artificially realised lines) with value $Z_{0}$. At the centre frequency, the insertion phase is $\varphi$ for LTL and $-\varphi$ for RTL where $\varphi$ is a positive phase angle. Four transmission lines of the same type can be connected as shown in Figure 1 to form either a 4-port unit cell (with ports 1, 2, 3 and 4), or a 5-port unit cell (with additional port 5 connected to the central node). The insertion phase between any two of ports $1,2,3$ or 4 is $2 \varphi$ for $X=L$, and $-2 \varphi$ for $X=R$. For the 5 -port case, of the insertion phase between port 5 and ports $1,2,3$ and 4 is $-\varphi$ for $\mathrm{X}=\mathrm{R}$.

Figure 2 shows the schematic of a 20-way power divider but the concept can be extended to $\mathrm{N}$-way subject to $\mathrm{N}=4 \mathrm{~m}$ where $\mathrm{m}$ is an odd integer. The power divider comprises a mosaic of unit cells as well as single transmission lines of type RTL or 
LTL. All the unit cells are 4-port except for the central unit cell which has 5-ports with its fifth port forming the input port (port 1) of the power divider. The unit cells marked with an $\mathrm{L}$ comprise transmission lines of type LTL $(\mathrm{X}=\mathrm{L})$ and those marked with an $\mathrm{R}$ comprise transmission lines of type RTL $(X=R)$.

An important feature of the power divider is that the $\mathrm{R}$ unit cells are connected only to L unit cells or transmission lines of type LTL, and vice versa for L unit cells. The significance is that all paths from the input port (port 1) to any output port (ports 2 to 21), there is zero insertion phase. Further, it can be shown that the voltages at the central node of each unit cell are equal in magnitude and phase, and are equal to that at the input and output ports. This is the infinite wavelength phenomena [6] in twodimensions. This also means that there is equal power division, and for the output ports terminated in matched loads (with impedance $Z_{o}$ ), the divider input impedance is $Z_{0} / N$.

\section{Simulation:}

We considered the simulation of a 20-way power divider as shown in Figure 2. $Z_{0}$ was chosen to be $100 \Omega$, and $\varphi$ was set to $5.65^{\circ}$ at $1 \mathrm{GHz}$. Conventional transmission lines were used to implement transmission lines of type RTL. The transmission lines of type LTL were artificially realised as a composite of conventional transmission lines (host medium), and lumped series capacitance and lumped shunt inductance similar to reference [7]. For both cases, the conventional transmission lines were of similar (but not identical) lengths and means that both $\mathrm{L}$ and $\mathrm{R}$ unit cells can have similar dimensions being about $8 \mathrm{~mm}$ by $8 \mathrm{~mm}$ when air-filled conventional transmission lines are used. The values of the lumped elements are comparable to values that have been used in practice [7]. 
Figure 3 shows the frequency responses of the key S-parameters such as the input reflection coefficient $\left(\mathrm{S}_{11}\right)$ and transmissions from the input port to the output port $\left(\mathrm{S}_{\mathrm{k} 1}\right.$, $\mathrm{k} \neq 1$ ). Due to symmetry it is only necessary to show transmissions $\mathrm{S}_{21}, \mathrm{~S}_{31}$ and $\mathrm{S}_{41}$. In the simulations, the reference impedance for port 1 was $5 \Omega$ and $100 \Omega$ for ports 2 to 21 . It can be seen that the power divider works as expected: matched input port and equal power division with equal phase. The slight de-tuning is due to inherent residual mismatch of the artificially realised left-handed lines. The input return loss is greater than $10 \mathrm{~dB}$ over a range of $600 \mathrm{MHz}$ (or $60 \%$ bandwidth). Over this band, the power division varies by only $0.6 \mathrm{~dB}$ with tracking better than $0.15 \mathrm{~dB}$ and $15^{\circ}$. Using only conventional right-handed transmission media, equal phase and equal power division could not be achieved to this level for a square shaped geometry. Moreover, a circular geometry such as that used in a radial power divider would be required [1].

\section{Conclusion:}

In this letter we have shown a square shaped $\mathrm{N}$-way power divider that essentially comprises a meta-material mosaic of unit cells that exhibit either left-handed or righthanded transmission behaviour. To achieve equal magnitude and phase power division for a square mosaic, left-handed unit cells are connected only to right-handed unit cells or right-handed transmission lines and vice versa. The resulting structure exhibits infinite wavelength phenomena in two dimensions. Simulation of a 20 -way power divider demonstrated the viability of the approach. The left-handed unit cells and transmission lines were artificially realised from a composite of conventional transmission lines and lumped elements. Since the power divider is square shaped, the output ports are located on straight lines and this allows ease of integration with power amplifier modules in a parallel combined power amplifier. 


\section{References:}

[1] A. E. Fathy, S.-W. Lee and D. Kalokitis, “A simplified design approach for radial power combiners", IEEE Trans. on Microwave Theory and Techniques, Vol. 54, No. 1, pp 247 - 255, Jan. 2006.

[2] H. Okabe, C. Caloz and T. Itoh, "A Compact Enhanced-Bandwidth Hybrid Ring Using an Artificial Lumped Element Left-Handed Transmission-Line Section", IEEE Trans. on Microwave Theory and Techniques, Vol. 52, No. 3, pp 798 - 804, March 2004.

[3] I-H. Lin, M. DeVincentis, C. Caloz and T. Itoh, "Arbitrary Dual-Band Components Using Composite Right/Left-Handed Transmission Lines”, IEEE Trans. on Microwave Theory and Techniques, Vol. 52, No. 4, pp 1142 - 1149, April 2004.

[4] R. Islam and G. V. Eleftheriades, "Phase-Agile Branch-Line Couplers Using Metamaterial Lines", IEEE Microwave and Wireless Component Letters, Vol. 14, No. 7, pp 340 - 342, July 2004.

[5] D. Kuylenstierna, S. E. Gunnarsson and H. Zirath, "Lumped-Element Quadrature Power Splitter Using Mixed Right/Left-Handed Transmission Lines", IEEE Trans. on Microwave Theory and Techniques, Vol. 53, No. 8, pp 2616 - 2621, Aug. 2005.

[6] A. Lai, K. M. K. H. Leong and T. Itoh, “A Novel N-Port Series Divider Using Infinite Wavelength Phenomena”, 2005 IEEE MTT-S Digest, pp 1001 - 1004.

[7] G. V. Eleftheriades, A. K. Iyer and P. C. Kremer, "Planar Negative Refractive Index Media Using Periodically L-C Loaded Transmission Lines”, IEEE Trans. on Microwave Theory and Techniques, Vol. 50, No. 12, pp 2702 - 2712, Dec. 2002. 


\section{Figure Captions:}

Figure 1:

Schematic of a 4-port unit cell (Port 5 not present) or a 5-port unit cell (Port 5 present). $\mathrm{X}$ is either $\mathrm{L}$ or $\mathrm{R}$.

Figure 2:

Schematic of a 20-way power divider. Input port (port 1) is located on the central unit cell.

Figure 3:

Calculated S-parameter frequency response.

$\rightarrow\left|S_{11}\right|-S_{21} \quad--S_{31} \quad-\cdots-S_{41}$ 
Figure 1:

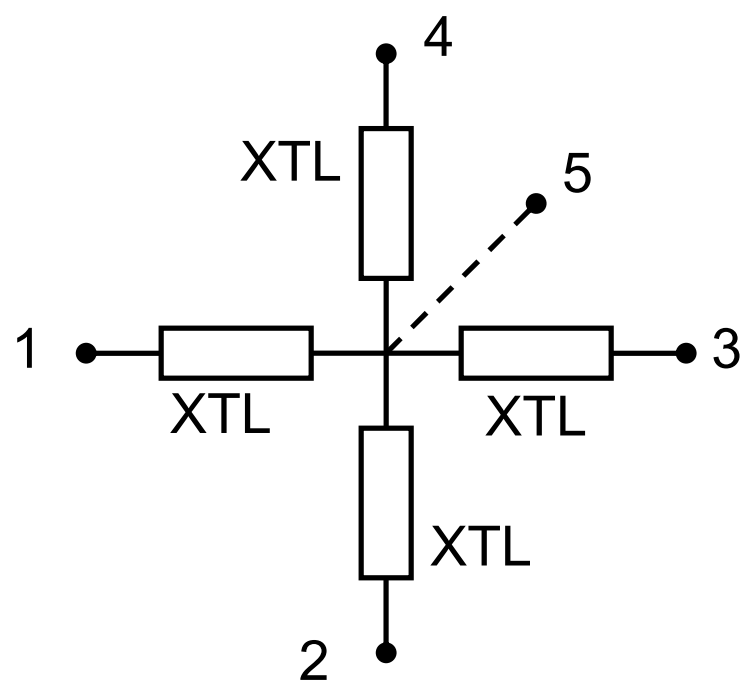


Figure 2:
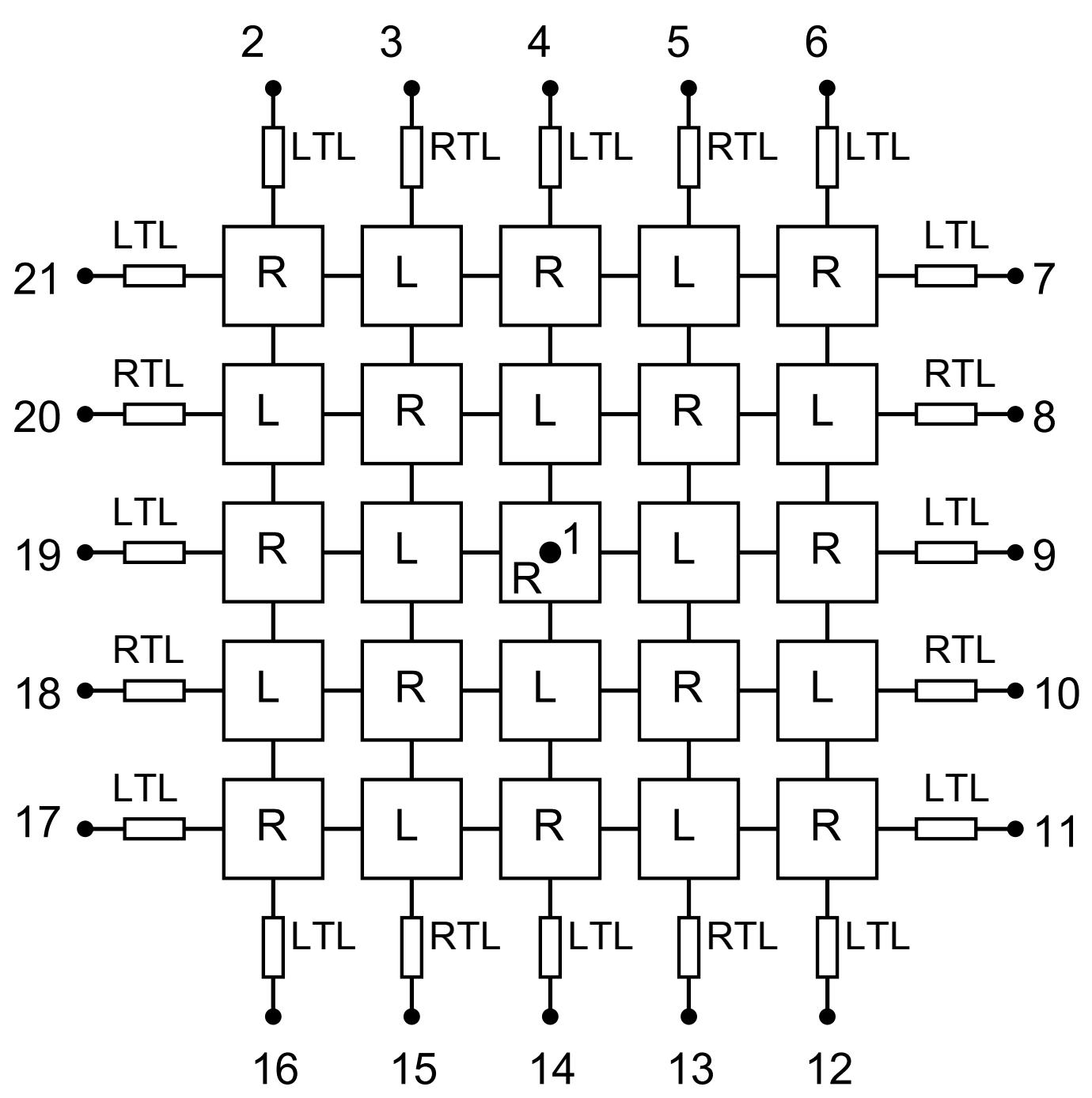
Figure 3:

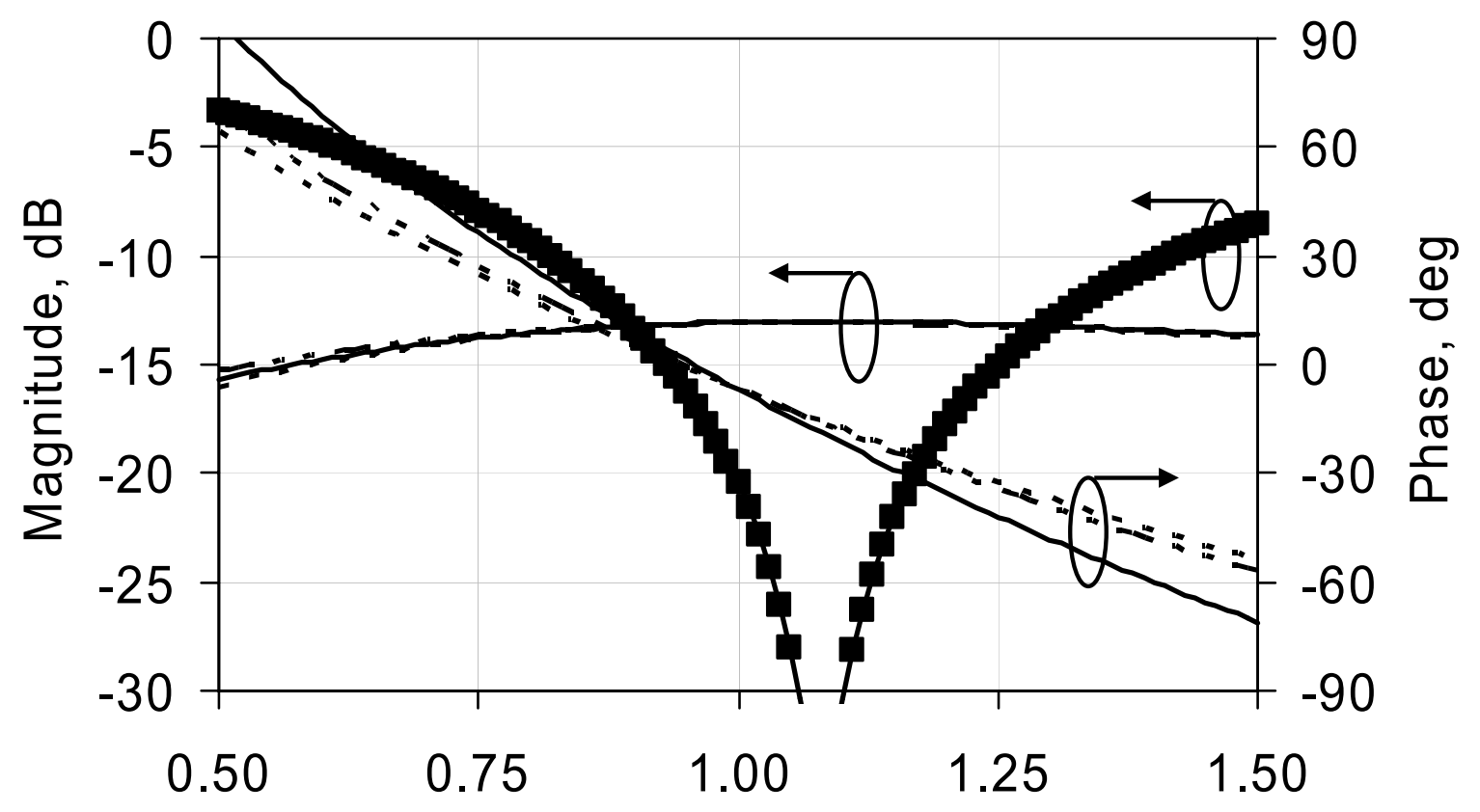

Frequency, $\mathrm{GHz}$ 